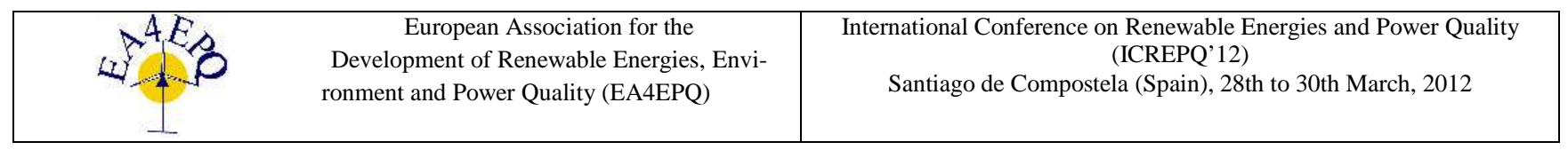

\title{
A Mathematical Method of Energy Resources Flows Data Validating Using the State Estimation Theory
}

\author{
Vladislav O. Samoylenko, Andrew V. Pazderin \\ Department of Automated Electrical Systems \\ Boris Yeltzin Ural Federal University \\ Mira, 19, 620002, Ekaterinburg (Russia) \\ Phone/Fax number: +007343 3754875, e-mail: vedmed@e1.ru, pav@daes.ustu.ru
}

\begin{abstract}
The energy efficiency improvement is known to start with creating the hi-technology energy resources metering and accounting system which enables the financial mutual settlements between the parties to be improved. With data errors or data loss due to any process failures, the financial consequences may turn out to be severe. As a rule, currently existing methods for energy billing imply a posteriori detection of gross errors in the measurements, which often leads to heated arguments of the parties involved and sometimes results in litigation. The paper presents a mathematical method for monitoring the validity of metering data in the energy transportation networks. The method proposed in the paper gives an opportunity to improve the reliability of measurements of energy, to smoothen inaccurate measurements and slight inconsistencies. Also, it provides for a bad data filtering at the stage of data acquisition. Taking into account developing the production infrastructure and increasing attention being paid to energy efficiency, the methodology is very promising.

Key words - data validation, energy efficiency, electric energy metering, energy flow problem, state estimation.
\end{abstract}

\section{Introduction}

The exploration of the Arctic and other areas by the world community is aimed primarily at developing the power industry in these areas. Nowadays, the issues of energy efficiency play a predominant role in all fields of the power industry. The energy efficiency improvement is known to start with creating the hi-technology energy resources metering and accounting system which enables the financial mutual settlements between the parties to be improved. Currently, automated metering systems for energy resources control and accounting (AMR) are being created.

The main framework for most types of energy transporting is a developed network infrastructure. Pipelines, heating mains and networks as elements of this infrastructure make the oil and gas industry as well as the heat and electrical power engineering industries similar. This results in the mathematical models used in research in these fields being also similar, while the physical essence of the processes remains different. The system of the energy resources transportation can be represented as a graph, with nodes being producers or consumers and branches being the mains (transmission lines, pipelines or heat mains).

AMR is expected to be able to recognize invalid data and restore the information lost. The paper presents a mathematical method for monitoring the validity of metering data in the energy transportation networks. This method has been inspired by the state estimation theory and serves as a practical application of energy flow problem [1].

\section{Theoretical background}

In an arbitrary network including a number of nodes and branches, there are nodal injections of energy from producers (electrical energy, a heat-transfer agent, a gas or solid fuel flow), the energy resource consumption 
in demand nodes, overflows in branches (i.e. power lines or pipelines) and losses (Figure 1).

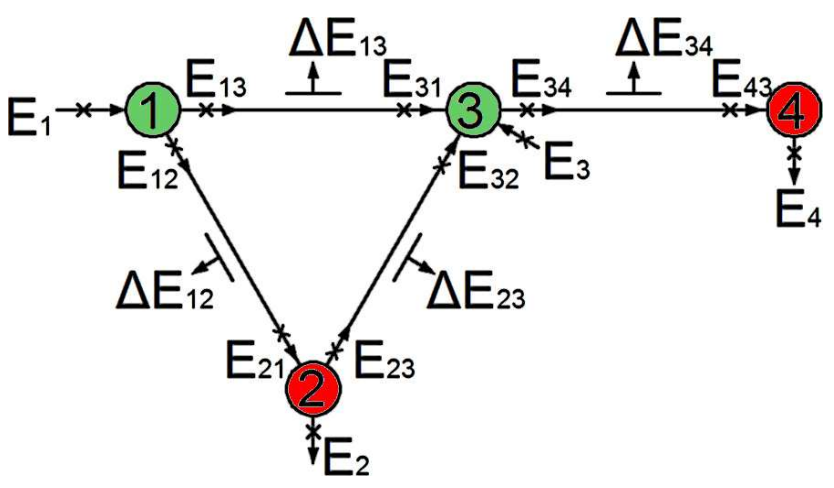

Fig 1. Energy transportation network

The energy resource losses in branches depend upon the specific features of a particular type of the energy resource and a physical principle of the transfer process.

In Figure 1, the symbol " $\mathrm{X}$ " denotes the presence of a metering system of an energy resource and the place of its location.

There is an energy resource flows balance at any moment. The equations of the balance can be represented as follows:

$$
\sum_{j \in U_{i}} E_{i j}+E_{i}=0, \quad i=1,2, \ldots, N,
$$

where $E_{i}$ is a nodal injection or consumption in node $i$; $E_{i j}$ is energy resource flows in branches connected to node $i ; \boldsymbol{\omega}_{i}$ is a set of nodes incidental (incidentalслучайный, несистематичный; adjacent-смежный, соседний, прилегающий, граничный) to $\dot{E}$ node; $N$ is the number of nodes.

Similarly, the balance equations for the branches are:

$$
E_{i j}+E_{j i}-\Delta E_{i j}=0, \quad i=1,2, \ldots, M
$$

where $E_{i j}$ is flows at the beginning of the branches; $E_{j i}$ is flows at the end of the branches; $\boldsymbol{\Delta} \boldsymbol{E}_{\mathrm{ij} j}$ is losses in the branches; $M$ is the number of branches.

Any measurement system has a certain error, so the balances based on the measurements are not satisfied in the nodes. Also, the values of measured flows are not identical to the caclulated ones. The energy measurement coordination is aimed at minimizing the sum of squares of relative measurements errors:

$$
F=\sum_{i=1}^{K} r_{i}\left(\frac{E_{i}^{\text {meas }}-E_{i}^{\text {calc }}}{E_{i}^{\text {meas }}}\right)^{\mathbf{z}} \rightarrow \min .
$$

where $E_{i}^{\text {meas }}$ is measured values; $E_{i}^{\text {calc }}$ is calculated values; $r_{i}$ is weight factors which are inversely proportional to the accuracy $\delta_{i}$ of the measurement system:

$$
r_{i}=\frac{1}{\left(\delta_{i}\right)^{2}}
$$

Energy measurement coordination is a typical nonlinear programming problem to be solved by appropriate methods [2].

As a matrix, the system of equations for all the measurements can be represented by:

$$
\mathbf{H} \cdot \mathbf{E}_{\mathbf{i}}^{\text {calc }}=\mathbf{E}_{\mathbf{i}}^{\text {meas }},
$$

where $H$ is an observability matrix. $\boldsymbol{E}_{i}^{\text {meas }}$ dimension is set by $\mathrm{K}$, and $E_{i}^{\text {calc }}$ dimension is set by M. Depending on the dimensions matching, there can be distinguished three different cases.

The first case deals with $K<M$, matrix $H$ being rectangular, there is an infinite set of uncertain solutions. The lack of measurement data results in energy resource flows distribution being impossible to obtain.

The second case deals with $K=M$, matrix $H$ being square, there is the only solution. Nevertheless, it is impossible to recognize the measurement errors and mitigate their impact.

In the case of $K>M$, matrix $H$ is rectangular, the solution is determined in terms of the energy flow problem. The measurement data is redundant. The solution of the redefined equation set (5) can be obtained by transforming it into a regular form equation set:

$$
\left(\mathbf{H}^{\mathrm{T}} \cdot \mathbf{R}^{-\mathbf{1}} \cdot \mathbf{H}\right) \mathbf{E}_{\mathrm{b}}^{\text {calc }}=\mathbf{H}^{\mathrm{T}} \cdot \mathbf{R}^{-1} \cdot \mathbf{E}^{\text {meas }}
$$

where $\mathbf{R}^{-1}$ is a diagonal weight ratio matrix, the dimensions of which are equal to the number of measurements $\mathrm{K}$. According to (4), the diagonal elements of the given matrix are defined as follows:

$$
r_{i i}=\frac{1}{\left(\delta_{i}-E_{i}^{\text {meas }}\right)^{2}}
$$

The conditions of observability, connected with topology of an energy transportation network and placing of metering systems, are defined as topological observability [1]. In the state estimation theory it is proved that the observability of the scheme is to be ensured by the number of measurement systems being not less than the number of branches $M$ in the scheme. The conclusion is of a great interest in terms of determining the proper placement of AMR in the system [3].

The observability criteria can be formulated for any number of energy meters with their random allocation in the network. The total number of variables in the linear equation system (1) and (2) is $N+M$. If all the doubling measurements relating to the beginning or to the end of the branch are ignored, the number of measurements will be $\vec{K}$. The general number of indeterminate flows in the scheme is always equal to $N+M-\vec{K}$. The equations of energy balances for branches and nodes can be represented as the reformed set of equations: 


$$
\mathbf{S}^{*} \cdot \mathbf{E}=\underline{\mathbf{E}},
$$

where $\mathbf{S}^{*}$ is a matrix, received as a result of transformation of a matrix of incidence $\mathbf{S}$; $\mathbf{E}$ - vector of indeterminate flows of an energy resource in branches and nodes of the network; $\underline{\mathbf{E}}$ - vector of measured flows of an energy resource in branches and nodes of the network. The observability matrix $\mathbf{H}=\mathbf{S}^{*}$ in many cases.

Dimension of vector is equal to the number of indeterminate flows of energy resource in the branches and nodes of the network, i.e. $N+M-\widetilde{K}$. Condition of finding the solution of equations set determines the sufficient condition of the observability:

$$
\operatorname{rank}\left(\mathbf{S}^{*}\right)=N+M-\vec{K} \text {. }
$$

As a rule, energy meters are installed in the nodes of an energy transportation network. The minimum measurement number ensuring the observability is $N-1$. The loop energy transportation network, where $M>N$, must be better provided with measurements for the state estimation.

Figure 2 shows the loop network with the nodal measurements only. For the purpose of simplification the losses are neglected.

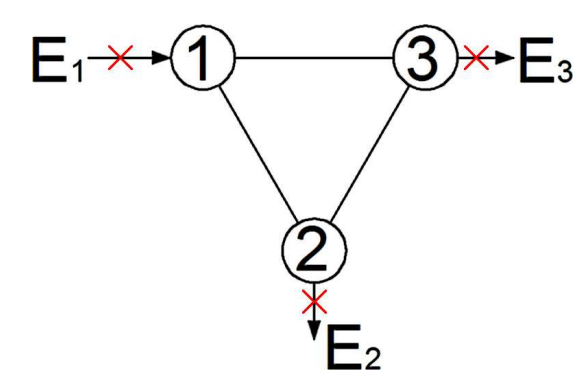

Fig 2. The unobservable network

The system of equations is presented:

$$
\begin{aligned}
E_{12}+E_{13} & =E_{1} \\
E_{12}-E_{23} & =E_{2} \\
E_{13}+E_{23} & =E_{3}
\end{aligned},
$$

or

$$
\left(\begin{array}{ccc}
1 & 0 & 0 \\
1 & -1 & 0 \\
0 & 0 & 1
\end{array}\right) \times\left(\begin{array}{c}
E_{12} \\
E_{2} \\
E_{3}
\end{array}\right)=\left(\begin{array}{c}
\underline{E}_{1}-E_{13} \\
\underline{E_{23}} \\
\underline{E_{13}}+\underline{E_{23}}
\end{array}\right)
$$

The unmeasured flows are in the left part of the system (10), and the measured flows are in right part accordingly. The measured energy resource flows are underlined. The $\mathbf{S}^{*}$-matrix rank is equal to: $\operatorname{rank}\left(\mathbf{S}^{*}\right)=2$. The unknown energy recources flows number is equal to $N+M-\widetilde{K}=(3+3)-3=3$. So, the observability condition (9) is unrealized - $2 \neq 3$, and the test network, presented in figure 2 , is unobservable. It is impossible to calculate energy resources flows because of the measurements absence inside the circuit (in network branches).

The variant including measurements in the branches instead the nodal measurement is presented in figure 3 . The branches 1-2, 2-3 and 1-3 are the independent circuit of electrical network.

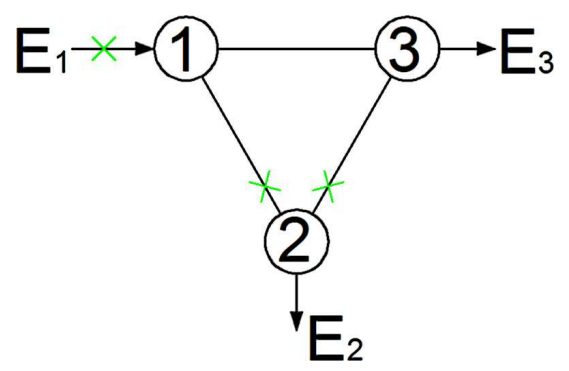

Fig 3. The observable network

The system of equations (11), according to (8), will be:

$$
\begin{aligned}
E_{12} & =E_{1}-E_{13} \\
E_{12}-E_{2} & =E_{23} \\
E_{3} & =\underline{E}_{13}+\underline{E}_{23},
\end{aligned}
$$

or

$$
\left(\begin{array}{ccc}
1 & 0 & 0 \\
1 & -1 & 0 \\
0 & 0 & 1
\end{array}\right) \times\left(\begin{array}{c}
E_{12} \\
E_{2} \\
E_{3}
\end{array}\right)=\left(\begin{array}{c}
E_{1}-E_{13} \\
E_{23} \\
E_{13}+\underline{E}_{23}
\end{array}\right)
$$

The $\mathbf{S}^{*}$-matrix rank is equal to: $\operatorname{rank}\left(\mathbf{S}^{*}\right)=3$. The right part of condition (9) is equal to: $N+M-\vec{K}=(3+3)-3=3$. So, the observability condition (9) is realized $(3=3)$ and the electrical loop network is observable. Using measured flows in node 1 and in the branches, it is possible to calculate other unmeasured energy resources flows in all the network, presented in figure 3 .

\section{An example}

An example of the state estimation is shown in Figure 4 (next page). The initial measured data is shown as the numerators of the fractions. At the left of the figure, there are the total values of an energy resource flows in the branches at different levels of the scheme. Due to the fact that AMRs contain certain errors, the balances of energy resource in the circuit are not met. Also, a comparison of inflows and outflows results in a conclusion which is contradicting to the law of conservation of energy and common sense. In solving the problem, smoothed data is obtained, and the balances are achieved (that is evinced by the numbers in the denominator; the difference in percent from the baseline is shown). Such data is suitable to be used in the further calculations. With the calculated data concerning the technological losses being available, the 
difference between actual losses and the technological ones will be commercial losses [4].

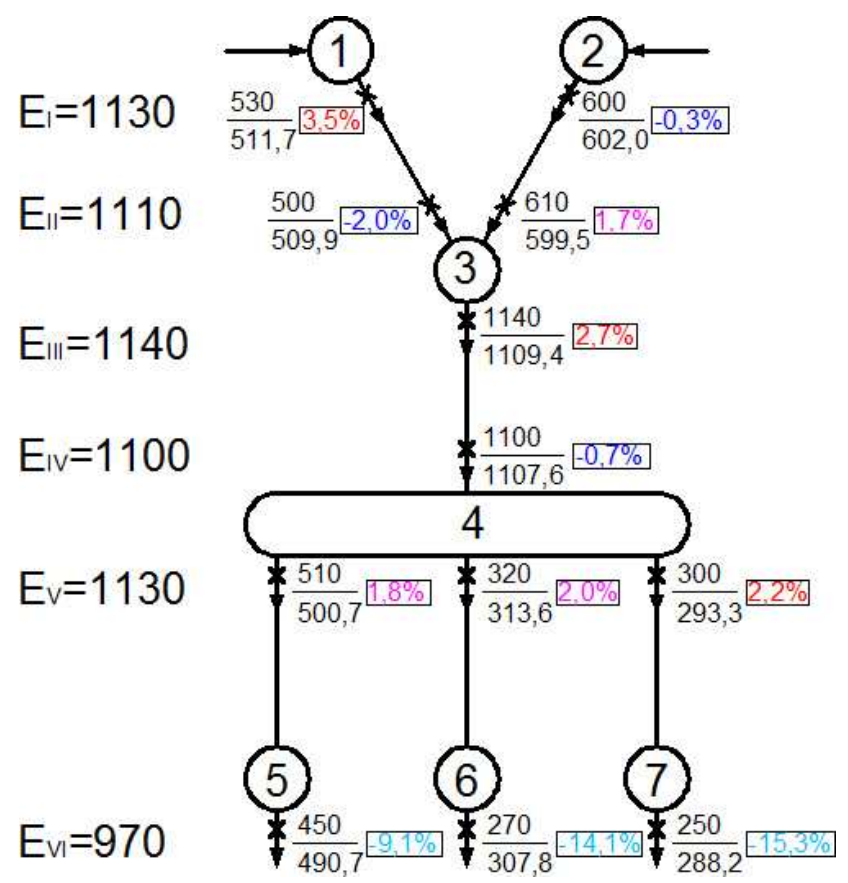

Fig 4. The test circuit

In addition, one of the major problems is that of the bad data identification. In case of the measurements containing small errors, which are the regular error of a measurement system, the financial effect may be negative for one of the parties, although not critical. With considerable errors or data loss due to any process failures, the consequences may turn out to be severe. As a rule, currently existing methods for energy billing imply a posteriori detection of gross errors in the measurements, which often leads to heated arguments of the parties involved and sometimes results in litigation. The validation method provides for a bad data filtering at the stage of data acquisition [5].

\section{Conclusions}

The method proposed in the paper gives an opportunity to implement a number of useful functions of prospective metering systems.
1. To improve the reliability of measurements of energy (electricity, gas, oil, heat-transfer), the transportation of which is carried out by the network infrastructure. As a result, a unified automated measuring system for controlling and accounting energy resources will be created and the system components responsible for making calculations will be unified.

2. To select the best suitable points for installing energy measurement systems on the basis of the state estimation theory.

3. To smoothen inaccurate measurements and slight inconsistencies in the group of measurements in order to obtain the compliance.

4. To detect a bad data, ensuring the possibility of re-inquiring as well as identifying the source of false information and signaling a malfunction in the system. To assess the level of commercial losses.

The method presented can form the framework of the next-generation high-tech systems for measuring and controlling energy resources. Taking into account the beginning of Arctic exploration, developing the production infrastructure and increasing attention being paid to energy efficiency, this method is very promising.

\section{References}

[1] Solution of energy flow problem using state estimation technique. 35th Annual Conference of IEEE Industrial Electronics Society, Porto, Portugal, 2009, p.17501755.

[2] Pazderin A.V., Plesnyaev E.A. Analysis of the Computation Techniques for Energy Flow Problem Solving // Proceedings IEEE Conference «Computer as a Tool» (EURO 2005), Belgrade, Serbia \& Montenegro, 2005. pp. 589-593.

[3] Egorov. A.V. The placement of power measuring systems in networks based on the theory of observability, Ekaterinburg, 2007. (in Russian)

[4] Pazderin A.V. The localization of commercial losses of electricity based on the solution of the energy flow problem // Industrial power engeneering. 2004. №9. C. 6-20. (in Russian)

[5] Pazderin A.V. Methods of improving validation measurement information systems, // University news. Issues of power engineering. 2004. № 11-12. C. 79-87. (in Russian) 